\title{
Preliminary study on knowledge graph construction based on the coupling mechanism of urban disaster and emergency response
}

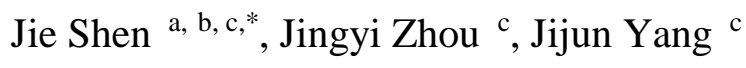 \\ ${ }^{a}$ Key Laboratory of Virtual Geographic Environment (Nanjing Normal University), Ministry of Education, Nanjing 210023, China; \\ shenjie@njnu.edu.cn (J.S) \\ ${ }^{b}$ Jiangsu Center for Collaborative Innovation in Geographical Information Resource Development and Application, Nanjing \\ 210023, China; \\ ${ }^{c}$ School of Geography, Nanjing Normal University, Nanjing 210023, China; 191302062@njnu.edu.cn (J.Z), chloeyangxx@163.com \\ $(J . Y)$ \\ * Corresponding author
}

Keywords: urban disaster, emergency response, coupling mechanism, knowledge graph

\begin{abstract}
:
According to the United Nations, more than half of the world's population lived in urban areas for the first time in 2008, towns and cities will be home to almost 5 billion people by 2030. Majority of the world's cities highly exposed to disasters, some large cities are exposed to as many as four or five different types of natural disasters, these cities are home to 1.4 billion people or around one third of the world's urban population. Urban disaster has become an important factor restricting regional modernization and sustainable development of social economy. Under the action of natural factors, human factors or both, it often causes temporary or long-term adverse effects on urban residents' life or social development. Urban disaster emergency response has become an important issue for governments, community organizations and even ordinary citizens around the world.
\end{abstract}

Scholars have carried out a lot of theoretical and applied research on urban disaster management and emergency response, including disaster mechanism model (Grassley, 2008; Gironás, 2010), disaster risk assessment (Carreño, 2017; Rus, 2018), emergency response decision making (Chiheb, 2019), and the emergency response ontology construction based on the emergency plan process (Amailef, 2013). Urban disasters occur in different disaster pregnant environments and have different impacts on urban spatial emergency response elements. However, there are few studies on the coupling mechanism of urban disasters and emergency response. Knowledge graph is a kind of graph model to describe the relationship between knowledge and everything in the world. It can be applied to data analysis, intelligent search, and decision support. Furthermore, knowledge graph can establish the relationship between disaster and emergency response, and reveal the network relationship between different entities, data resources and disaster events.

Research on ontology and knowledge graph for some disasters such as urban waterlogging and epidemics has started (Pesquita, 2014; Chen, 2020; Wu, 2020), The current research trend is to extract entities, attributes and relationships from multi-source disaster and emergency response data, combine top-down and bottom-up to build disaster emergency ontology and knowledge graph, and support the deep integration of disaster emergency scene information.

This paper aims to solve the scientific problem of how to construct the ontology and knowledge graph based on the coupling mechanism of urban disaster and emergency response. It is mainly divided into three aspects:

1. Research on the coupling mechanism of urban disasters and emergency response. Study the causes and characteristics of the development of urban natural and man-made disasters, and explore the influence process of disasters on emergency response elements, Taking urban waterlogging as an example, according to the flood control emergency plan, indicators such as the depth of water waterlogging, the number of people affected, and economic losses can be obtained to determine the emergency response standards of the emergency department.

2. Formal representation of coupling mechanism of urban disasters and emergency response such as ontology construction, which plays an important role in building the conceptual framework of knowledge graph. It is mainly to collect the core concepts in the field of disaster and emergency response, organize the relationship between concepts, such as analyzing the disaster and emergency knowledge contained in disaster academic literature, emergency plans, and emergency cases. Finally, studying and summarizing the basic terms and concepts under the guidance of domain experts, which is a top-down process.

3. Knowledge graph construction for disaster and emergency response. Urban disaster and emergency rescue is usually participated by multiple users, taking urban waterlogging as an example, before the disaster, the meteorological departments forecast and monitor the waterlogging data through real-time sensors such as water meter and radar water level gauge, and judges the waterlogging level. During the disaster, the road traffic headquarters is responsible 
for organizing traffic dredging, emergency drainage, etc. After the disaster, the Emergency Management Bureau is responsible for flood control verification, loss assessment and other disaster relief work. Different disasters correspond to different emergency departments, rescue tasks, emergency data and evacuation models, knowledge graph can mine the relationship among different model parameters, entities and disaster events through network structure, which is a bottom-up process.

We plan to solve these problems in following aspects:

1) According to different types of urban disasters, the corresponding disaster mechanism, causes and processes are studied, and then combined with the analysis of emergency response process, the correlation analysis of disasters and emergency response elements is carried out. Furthermore, a new model is constructed by integrating the disaster calculation model with the emergency evacuation model.

2) Currently, there is no standardized process and method for building domain ontology, but the maturity of the more commonly used ontology building methods are: Seven step, Methontology, IDEF5, TOVE, Skeleton, SENSUS and KACTUS. The process of ontology construction includes listing the important concepts or terms, definition classes and the classification system, attributes and relationships of classes in urban disaster and emergency response domain, and then realizes the ontology implementation, evaluation, correction and storage.

3) Knowledge graph construction of urban disaster and emergency response, Firstly, the knowledge base of model parameters in the field of disaster and emergency response is established. Then through natural language processing, machine learning, data mining and other artificial intelligence means to extract entities, attributes and relationships from multi-source heterogeneous data, include structured, semi-structured and unstructured data from different sources. Finally knowledge can be stored in two main ways: RDF store and Graph Database.

This study explores the coupling mechanism of urban disaster and emergency response, constructs the coupling model of disaster and emergency response, formalizes the relationship between disaster and emergency response through ontology method, and sorts out the concept and its relationship level. Knowledge graph provides a theoretical basis for big data integration of urban disaster and emergency response, supports the deep integration of disaster scene information at different stages, and provides accurate disaster emergency services.

Acknowledgements:

This research was funded by National Natural Science Foundation of China awarded project (No. 41871371).

\section{Reference:}

Amailef, K., \& Lu, J., 2013.Ontology-supported case-based reasoning approach for intelligent m-Government emergency response services. Decision Support Systems, 55(1), 79-97.

Carreño, M. L., Cardona, O. D., Barbat, A. H., Suarez, D. C., del Pilar Perez, M., \& Narvaez, L., 2017. Holistic disaster risk evaluation for the urban risk management plan of Manizales, Colombia. International Journal of Disaster Risk Science, 8(3), 258-269.

Chen, X., Liu, J., Xu, L. Zhang, W., \&Liu, H., 2020. Construction of the COVID-19 Epidemic Cases Activity Knowledge Graph:A Case Study of Zhengzhou City, Journal of Wuhan University (Information Science Edition), 816-825.

Chiheb, F., Boumahdi, F., \& Bouarfa, H., 2019. A new model for integrating big data into phases of decision-making process. Procedia Computer Science, 151, 636-642.

Gironás, J., Roesner, L. A., Rossman, L. A., \& Davis, J., 2010. A new applications manual for the Storm Water Management Model (SWMM). Environmental Modelling \& Software, 25(6), 813-814.

Grassly, N. C., \& Fraser, C., 2008. Mathematical models of infectious disease transmission. Nature Reviews Microbiology, 6(6), 477-487.

Pesquita, C., Ferreira, J. D., Couto, F. M., \& Silva, M. J., 2014. The epidemiology ontology: an ontology for the semantic annotation of epidemiological resources. Journal of biomedical semantics, 5(1), 1-7.

Rus, K., Kilar, V., \& Koren, D., 2018. Resilience assessment of complex urban systems to natural disasters: A new literature review. International journal of disaster risk reduction, 31, 311-330.

Wu, Z., Shen, Y., Wang, H., \& Wu, M., 2020. An ontology-based framework for heterogeneous data management and its application for urban flood disasters. Earth Science Informatics, 1-14. 